УДК 528.7:629.78

DOI https://doi.org/10.32838/2663-5941/2020.4/14

Мещеряков Д.В.

AO PETROSOFT (г. Одесса)

\title{
НЕЧИСЛОВАЯ ОБРАБОТКА ДАННЫХ В ИНФРАКРАСНОЙ СИСТЕМЕ С БИОЛОГИЧЕСКОЙ ОБРАТНОЙ СВЯЗЬЮ
}

Розглянуто обробку вихідної ознакової інформації в інфрачервоній системі пелоїдотерапії з біологічним зворотним зв'язком, яка може бути використана для лікування опорно-рухового апарату членів екіпажу судів. Обтрунтовано необхідність узгодження кібернетичного і фізіологічного підходів описів, що використовуються для технічних і біологічних об'єктів. Показано, що основними проблемами, які виникають під час обробки вихідних ознакових сигналів є їх нечіткість і неоднозначність. Ці особливості реакиії фізіологічного об'єкта на інфрачервоний вплив зумовлені як складністю фізіологічного об' 'кта, так і накладанням супутніх сигналів на інформачійні сигнали відносно управління прочесом опромінювання. Обгрунтовано складність одержання аналітичних зв'язків між вхідним впливом і відгуком організму, який може бути зафіксовано неінвазивними технічними засобами. Запропоновано для опису вихідних ознак використовувати методи статистики нечислових даних. Виявлено особливості обробки якісних даних відповідно до прийняття рішень для управління замкнутою системою регулювання інтенсивності випромінювання. Показано, що показником вибраних даних може служити середне по Кемені. Співставлення послідовності середніх значень ковзаючої вибірки дало змогу визначити механізм рішення стосовно направлення зміни інтенсивності інфрачервоного випромінювання, що генерується. Обтрунтовано доцільність використання в системі з біологічним зворотним зв'язком критерію знаків для прийняття рішень у темі з біологічним зворотним зв'язком. Досягнення локальних ицілей управління інтенсивністю опромінювання біологічного об'єкта зводиться до принципу стежачого урівноваження, для якого характерний високий ступінь стійкості до аномальних викидів. Для компенсачії недоліку, пов'язаного з великим часом виходу на режим регулювання, запропоновано два шиляхи підвищення динамічних характеристик управління. Перший засновано на нечислових методах по аналогії з роботою біологічних сенсорів, другий - на нечітких регуляторах.

Ключові слова: біологічний зворотний зв'язок, нецифрова статистика, прийняття рішень, управління.

Постановка проблемы. Системы автоматического управления базируются на кибернетических принципах, которые включают в себя: модельное представление объектов, их формальное описание, модульный принцип построения сложных систем, рассмотрение элементов в терминах «вход-выход-состояние». Замена реального объекта его моделью, для последующего экспериментирования с ней, предполагает, что при неизменном входном воздействии выходная реакция элемента будет повторяемой.

Введение биологической обратной связи в систему кардинально меняет ситуацию, поскольку к человеку, как к элементу системы управления, невозможно применить понятие линейности, а неизменное входное воздействие не дает повторяющейся реакции. Биологический объект настолько сложен, что стандартные математические методы описания, применяемые для технических объектов, малоэффективны. Данные методы используются для формализации систем с биологической обратной связью при условии, что объект может быть представлен некой обобщенной, т.е. кибернетической моделью. Примером может служить тепловая модель усредненного птенца в системе управления инфракрасным обогревом при промышленном выращивании цыплят.

Для биологических объектов более адекватной представляется физиологическая модель Анохина, для которой характерна взаимосвязь и взаимообусловленность составляющих подсистем и существенная зависимость выходных реакций от предыстории процесса и реализуемых подцелей. Сенсорные системы организма сложнее сенсоров технических систем, в них входят вспомогательные структуры, оптимизирующие работу рецепторов, которые не просто передают возбуждение, а адаптируются под характеристики раздражителя. Сенсорная система имеет обратные связи с последующими и предшествующими структурами, передача сигналов идет по разным 
каналам, обработка сенсорного возбуждения осуществляется не только в центральной нервной системе, но в нижележащих структурах. Следствием этого является различное время реакции организма на входное воздействие, что может влиять на устойчивость управляющей биотехнической системы.

Важную роль играет также распространение информации в физиологической системе. Каналы передачи имеют очень низкую пропускную способность по сравнению с техническими системами, однако обеспечивают устойчивое функционирование организма в реальном масштабе времени. Сигналы рецепторов имеют сложную структуру, содержат данные начала и окончания воздействия, амплитуда и частота в которых меняется по мере воздействия, подстраивают свою активность в ходе действия длительного раздражения. Очевидно, что ни частота биологических импульсов, ни амплитуда не способны нести в себе всю информацию относительно входного воздействия в силу их ограниченного диапазона. Вместе с тем известно сколь малые перепады воздействий идентифицируются физиологической системой и как велик динамический диапазон воспринимаемых воздействий. Организм выполняет множество функций для поддержания гомеостаза и баланса с окружающей средой. Все это приводит к тому, что невозможно выделить четкую и однозначную связь между входным воздействием и выходным признаком проявления этой реакции. Выходные признаки организма несут информацию смешанного типа, а если учесть, что инвазивные средства считывания неприемлемы для проведения физиологических процедур и следует ограничиться неинвазивными средствами, это приводит к еще большему снижению информативности.

Актуальность исследования процессов обработки данных в медицинских биотехнических системах не вызывает сомнения. Системы с биологической обратной связью получают все большее распространение, поскольку исключают фармакологические средства и актуализируют внутренние резервы организма в процессе проведения лечебных процедур. Совмещение кибернетического и физиологического подходов существенно повышает интеллектуальную составляющую биотехнических систем за счет использования преимуществ каждого компонента. Противоречие между кибернетическим и физиологическим подходами, различие в методах преобразования сигналов составляют глав- ную проблему создания биотехнических систем с биологической обратной связью.

В данной работе рассматривается подход к проблеме преобразования информации применительно к системе регулирования интенсивности инфракрасного излучения по физиологической реакции организма человека, находящегося в цепи биологической обратной связи [1]. Поскольку реакция человека на тепловое воздействие зависит от его массы, возраста, характера заболевания, то достижение условий индивидуального облучения позволяет оптимизировать лечебный процесс пелоидотерапии.

Анализ последних достижений и публикаций. Методы обработки биометрических сигналов в значительной мере определяются используемыми измерительными преобразователями, поскольку инвазивные или неинвазивные средства мониторирования оказывают существенное влияние на качество и информативность считываемых сигналов [2]. В работе [3] показано, что наиболее значимыми признаками, характеризующими тепловое воздействие на организм, являются частота сердечных сокращений и дыхания, температура кожного покрова и потовыделение. Предварительная обработка полезного сигнала из считываемого выходного сигнала вариабельности сердечного ритма осуществляется линейными спектральными методами [4] или методами нелинейной фильтрации [5], что позволяет выделять биологические сигналы на фоне сопутствующих сигналов. Эффективная информационная технология обработки сигналов включает представление модели полезного сигнала в виде решетчатой функции, разложение сигнала по базисным функциям, а также кусочной в виде последовательности фрагментов [6]. Основным ограничением таких подходов является тот факт, что сигналы биологического объекта воспринимаются однозначными, несущими информацию только относительного исследуемого процесса [7]. Вместе с тем проблема обработки биологических сигналов применительно к управлению техническими объектами далека от разрешения. Такие особенности сигналов, как нечеткость и неоднозначность относительно первичного возбуждения, усугубляют проблему [8]. Использование нечетких методов [9] позволяет в определенной мере снизить это влияние. Применительно к задачам управления в системах с биологической обратной связью проблема неоднозначности реакции на известное входное воздействие даже не рассматривалась [10]. При решении сложных задач 
принятия решений в экономике, социологии, математическом моделировании применяются методы нечисловой статистики [11]. Однако информация относительно возможности использования этих методов преобразования выходных признаковых данных биологического объекта в технико-биологических управляющих системах отсутствует.

Формулирование целей статьи. Целью работы является анализ возможности использования методов нечисловой статистики для преобразования выходных признаковых сигналов человека, находящегося в цепи биологической обратной связи системы управления интенсивностью инфракрасного облучения.

Для достижения этой цели необходимо решить задачи:

1) определить значимый признак выборки выходных признаков биологического объекта, обеспечивающий устойчивость обработки данных;

2) разработать алгоритм принятия решений для управления инфракрасными излучателями.

Изложение основного материала. Выходное информационное признаковое пространство биологического объекта носит вероятностный характер, что связано с низким уровнем выходных сигналов, взаимным наложением разнородных сигналов, шумом и влиянием внешних воздействий. Это предопределяет использование статистических методов обработки данных и учет того обстоятельства, что считываемая последовательность носит нестационарный характер. Нестационарность приводит к необходимости выделения квазистационарных участков, на которых возможно выделение моментов стационарной последовательности.

Кроме того, признаковые сигналы, например проводимости кожного покрова, полученные на различных участках тела, имеют существенный разброс. Это связано с неравномерным расположением по поверхности тела потовых желез, различной толщиной эпидермиса и т.п. Более того, прямой связи сопротивления кожного покрова и активизации потовых желез нет, а изменение сопротивления кожного покрова в два раза вовсе не означает, что их активность изменилась пропорционально. Отсюда следует, что выходные данные несут информацию относительно физиологических процессов в организме, однако эти данные являются скорее качественными, чем количественными.

Такими качественными уровнями в системе инфракрасной пелоидотерапии могут служить проницаемость кожного покрова для лечебного пелоида, которая условно описываются пятью уровнями: непроницаемого, плохо проницаемого, средне проницаемого, проницаемого, хорошо проницаемого [12]. Данные состояния представляются сопротивлением кожного покрова, значения которого лежат в диапазоне от единиц МОм до сотен Ом. Очевидно, что сопротивление кожного покрова не эквивалентно активности потовых желез, поэтому привязка физических показаний прибора к физиологической проводимости пелоида является качественной, а не количественной.

Качественная природа выборок, в отличие от количественного представления, имеет свои особенности. Так, значения выборок нельзя складывать, поскольку полученные значения при этом теряют содержательный смысл. Если предположить, что последовательность выборок $x_{1}, x_{2}, \ldots, x_{n}$ являются элементами нелинейного множества $X$, то при приведенных ограничениях становится понятным, что определение среднего значения выборки требует иных подходов по сравнению с принятыми. Даже при анализе выборок цифрового ряда среднеарифметическое значение применимо только для случая достаточно равномерного значения членов ряда. Если в совокупности выборки присутствует аномальное значение, то величина среднеарифметического значения выборки не всегда адекватно характеризует среднее, поскольку влияние данной составляющей гораздо значимее остальных.

В нецифровой статистике мерой различия служит показатель $d: X^{2} \rightarrow[0,+\infty]$, заключающийся в фиксации того факта, что чем больше $d(x, y)$, тем более разнятся $x$ и $y$ [11]. Применительно к эмпирическому среднему это означает минимизацию выражения:

$$
E_{n}(d)=\operatorname{Arg} \min \left\{\sum_{1 \leq i \leq n} d\left(x_{i}, x\right), x \in X\right\},
$$

где среднее $E_{n}(d)$ представляет совокупность $x \in X$, для которых функция

$$
f_{n}(x)=\frac{1}{n} \sum_{1 \leq i \leq n} d\left(x_{i}, x\right)
$$

достигает минимального значения на множестве $X$ и является медианой или средним для выборки ранжировок по Кемени. В [11] показано, что для качественных значений для порядковой шкалы в качестве среднего можно использовать только медиану, а не среднеарифметическое или среднегеометрическое. Доказательство сходимости теоретического и эмпирического 
средних основывается на законе больших чисел. При ограниченной выборке вводится понятие $\varepsilon$-пятки $f$, которая представляет собой окрестность $\operatorname{Arg} \min (f)$ в терминах минимизируемой функции. Этим, в частности, снимается вопрос о выборе метрики в пространстве $X$. Размеры $\varepsilon$-области определяются как точностью определения значений, так и с помощью используемых порогов чувствительности, если модуль разности между выборками меньше или равен порогу чувствительности.

Из особенностей качественного представления следует также и то, что увеличение объемов выборок может не привести к росту состоятельности оценки, поскольку невозможно говорить о стационарности и центрированности анализируемого процесса. Наряду с негативными следствиями такого утверждения имеет место и позитивная сторона - малый объем выборки и, соответственно, время выборки. Это обстоятельство критично для систем управления, поскольку вносит запаздывание, которое составляет не менее половины времени выборки. Малый объем выборки приводит к значительному разбросу показаний относительно среднего, поскольку для систем управления важен показатель устойчивости. Если рассматривать устойчивость как отсутствие выхода управляющих воздействий относительно допустимых отклонений, то реакция биологического объекта вида «холодно - тепло» или «комфортно - не комфортно» более устойчиво, чем восприятие цифрового значения температуры среды. Ощущение тепла каждым человеком воспринимается индивидуально, физиологическая реакция организма первична, а количественное описание условий вторично.

Шкалами качественных признаков являются порядковая шкала и шкала наименований [11], первая из которых соответствует решаемой задаче управления. Сравнение двух выборок $Y$ и $Z$ можно осуществить по их средним значениям:

$$
f\left(Y_{1}, Y_{2}, \ldots, Y_{n}\right)<f\left(Z_{1}, Z_{2}, \ldots, Z_{n}\right) .
$$

Если допускаются преобразования в порядковой шкале $\rho$, например нормирование, то $Y_{i}$ и $Z_{i}$ меняются на $\rho\left(Y_{i}\right)$ и $\rho\left(Z_{i}\right)$.

Для формирования среднего из совокупности данных можно использовать признак расстояния от данной точки до точек окружения, причем мерой близости являются меньшие расстояния. Поскольку для качественных значений невозможно использование операции суммирования, используем показатель различия. Для задач управления с ограниченной выборкой необходимо определение эмпирического среднего, которое при определенных условиях обеспечивает сходимость с теоретическим средним.

Для пространства произвольной формы $X$ с элементами $x_{1}, x_{2}, \ldots, x_{n}$ действительнозначной функции $f(x, y)$ со значениями в $X$, значения функции различия $f(x, y)$ отличаются тем больше, чем более различаются $x$ и $y$. Средней величиной $\bar{x}$ относительно меры различия $f(x, y)$ является решение оптимизационной задачи [11]:

$$
\sum_{i=1}^{n} f(x, y) \rightarrow \min , \quad y \in X .
$$

Теоретическое среднее не отличается от классического среднего для закона больших чисел при $n \rightarrow \infty$, в соответствии с теоремой Хинчина, стремится к математическому ожиданию:

$$
\frac{1}{n} \sum_{i=1}^{n} f\left(x_{i}, y\right) \rightarrow M f(x, y) .
$$

При $f(x, y)=|x-y|$ и нечетном количестве выборок $n=2 k+1$ значение среднего равно $\bar{x}=x_{k+1}$, т. е. получаем выборочную медиану. При четном количестве членов выборки получаем полусумму выборочных значений $x_{k}$ и $x_{k+1}$. Для исключения арифметических действий можно ограничиться нечетным количеством выборок.

Для определения среднего по Кемени необходимо провести ранжировку данных. Для управляющей системы запаздывание определяется половиной длины выборки, поэтому она не может быть большой. При ограниченной выборке операция ранжирования состоит в расположении данных выборки в порядке неубывания, т.е. возрастания с возможностью существования элементов с одинаковыми значениями. Алгоритмы реализации такой функции известны и состоят в последовательном сравнении текущего элемента из выборки с выстроенными в порядке возрастания элементами. Далее определяется медиана, которая и является средним по Кемени.

Качественный характер выборок приводит к непараметрическим моделям описания процессов. Параметрическая вероятностно-статистическая модель представляется вектором фиксированной размерности, которая не зависит от объема выборки. В непараметрических моделях неприемлемо понятие плотности распределения, поэтому его можно заменить на вероятность попадания в $\varepsilon$-область. Формирование $\varepsilon$-области в простейшем случае 
может быть установкой по уровню шума в обрабатываемом сигнале, требуемой чувствительности или иным критериям. В начальной стадии можно предусмотреть вариант асимптотического приближения к цели регулирования, затем уточнять в зависимости от существующих ограничений. Такое решение позволяет говорить о состоятельности предложенного подхода.

Для решаемой задачи управления интенсивностью инфракрасного излучения человека по его физиологическим признакам важно то, что устойчивость определения физиологической реакции выше, чем числовых величин приборов, поскольку она первична. Очевидно, что использование, кроме сопротивления кожного покрова, иных признаков реакции человека на инфракрасное облучение сохраняет качественный характер этих сигналов. Так, частота сердечных сокращений и дыхания лишь косвенно отражает факт усиления отбора тепла периферийной сосудистой и дыхательной системой.

Согласование функциональной и кибернетических структур в биотехнической системе управления с биологической обратной связью может быть достигнуто путем определения общих свойств данных систем. В биологическом объекте ответ на каждую реакцию сопровождается формированием элементарной цели, осуществлением деятельного акта, проверкой ее достижения, корректировкой элементарной цели и так далее. Эти действия происходят в рамках цели более высокого уровня, например поддержание температурных условий функционирования организма. Естественно, что это крайне упрощенная модель. Однако она может служить основой для согласования кибернетического и функционального подходов.

Выборка признаков $x_{1}, x_{2}, \ldots, x_{n}$ считываемой случайной величины $X$ биологического объекта в силу отмеченных выше причин не может иметь известную функцию распределения $F(x)$. При увеличении объема выборки, в соответствии с центральной предельной теоремой, функция распределения стремится к нормальному закону распределения. Для непараметрической модели наиболее соответствующими решаемой задаче управления являются два критерия: критерий знаков и критерий знаковых рангов [13]. Для критерия знаков $F(m)=0,5$, т.е. каждая из случайных величин равновероятно больше другой выборки:

$$
R_{j}=\left\{\begin{array}{lll}
-1, & \text { если } & x_{j}<m_{0} \\
+1, & \text { если } & x_{j}>m_{0}
\end{array} .\right.
$$

Если величина $m_{0}$ соответствует отклику сенсора при активном потоотделении, то представленная связь отображает принятие решения в окрестности активной терапевтической зоны. Сигнал «-1» свидетельствует о том, что интенсивность излучения необходимо повысить, «+1»интенсивность излучения необходимо понизить, « $x_{j}=m_{0} »-$ оставить неизменным. Данный подход управления известен как принцип следящего уравновешивания. Основным достоинством следящего уравновешивания является высокая устойчивость преобразования при низком отношении сигнал/шум, недостатком - низкая скорость выхода на режим. Если последний недостаток не является принципиальным, например, за счет предварительного прогрева камеры пелоидотерапии перед проведением процедуры, то принятие решения относительно управления инфракрасными излучателями может ограничиться критерием знаков в представленном варианте.

Если важна скорость выхода системы на режим регулирования, можно воспользоваться методами нечеткой логики. Принятия решений относительно изменения интенсивности инфракрасного излучения по выходным признакам пациента, находящимся в цепи биологической обратной связи, относятся к нечетко определенным. Это обусловлено тем, что ощущение тепла для различных людей отличается, следовательно, и неоднородной является выходная признаковая реакция на это воздействие. Полагаем, что цель имеет множество альтернатив, причем априори считаем, что альтернативы равноправные, что соответствует подходу Беллмана-Заде.

Обозначим счетное количество альтернатив $a \in A$, где $A$ некоторое нечеткое множество, а нечеткое множество целей через $G \subset A$. Функцию принадлежности каждой конкретной альтернативы этому подмножеству представим в виде $\mu_{G}: A \rightarrow[0,1]$. Нечеткие ограничения представим множеством допустимых альтернатив $C$ из того же множества $A$ с функцией принадлежности $\propto_{C}$.

Задача сводится к достижению нечетко определенной цели $\propto_{G}$ при нечетко заданных ограничениях $\propto_{C}$. Решением является пересечение нечетких множеств $D=G \cap C$, функция принадлежности которого достигает $\mu_{D}=\min \left\{\mu_{G}(a), \mu_{C}(a)\right\}$.

При наличии нескольких целей $G_{1}, G_{2}, \ldots, G_{m}$ и ограничений $C_{1}, C_{2}, \ldots, C_{n}$ необходимо учитывать весовые коэффициенты значимости каждого достижимости целей $\alpha$ и ограничений $\beta$. Так, для задачи повышения проводимости кожного покрова значимость данных о сопротивлении 
кожного покрова существенно выше, чем частота сердечных сокращений и частота дыхания, которые важны только при значительных интенсивностях облучения поверхности человека. Функция принадлежности приобретает вид

$$
\begin{gathered}
\mu_{D}(a)=\min \left\{\alpha_{1} \mu_{G 1}(a), \ldots, \alpha_{m} \mu_{G m}(a),\right. \\
\left.\beta_{1} \mu_{C 1}(a), \ldots, \beta_{n} \mu_{C 1}(a), \ldots, \beta_{n} \mu_{c n}(a)\right\}
\end{gathered}
$$

Это означает, что чем больше степень принадлежности $\propto_{G}$ одной из альтернатив множеству цели $G(a)$, тем более высокой будет степень достижения этой цели при выборе данной альтернативы $a$ при решении данной задачи.

Возможен и биологический подход к повышению динамических характеристик следящей системы уравновешивания. Для этого воспользуемся упрощенной моделью формирования отклика от сенсора биологической системой (рис. 1) [12].

Кодирование информации в биологических анализаторах осуществляется как в амплитудной, так и в импульсной форме. При этом содержание информации определяется не только амплитудой импульсов, но и количеством импульсов в единицу времени, соединением импульсов в пачки, числом импульсов в пачке, интервалами между пачками. Такая сложная комбинация способов представления информации позволяет как повысить надежность передачи первичных сообщений в центральную нервную систему, так и обеспечить столь большой динамический диапазон воспринимаемых воздействий биологической системой. Очевидно, что ни один из отдельно взятых способов кодирования не в состоянии обеспечить передачу достоверной информации от сенсора при столь ограниченной пропускной способности биологического канала связи.

Таким образом, качественное представление выходных признаков биологического объекта, находящегося в цепи биологической обратной связи системы регулирования, позволяет формировать запросы на управление интенсивностью инфракрасного излучения по физиологической реакции организма. По выходной физиологической информации как реакции на внешнее тепловое воздействие, так и на внутреннюю адаптацию организма к собственным целям и исполняемым процессам осуществляется изменение внешнего воздействия с учетом неоднозначности преобразования.

Применительно к управлению интенсивностью инфракрасного излучения по одному из выходных признаков биологического объекта, например, сопротивлению кожного покрова, вариант решения задачи сводится к следующему:

1) преобразование выходного признака в бинарный сигнал;

2) выявление ограничений зоны признаков управления;

3) выделение терапевтической зоны управления;

4) качественное представление лепестков управления;

5) распределение интенсивности управления в зоне лепестков;

6) формирование управляющего воздействия.

Реализация представленного варианта управления интенсивностью инфракрасного нагрева пациента в камере инфракрасной пелоидотерапии выполнена на базе технологии ARDUINO. Ограничения интенсивности излучения инфракрасных излучателей выбирались из нижнего условия некомфортности нахождения в помещении. Этому условию соответствуют значения сопротивления кожного покрова выше 1 МОм. $\mathrm{C}$ точки зрения проведения процедуры диапазон

\section{Слабый раздражитель}

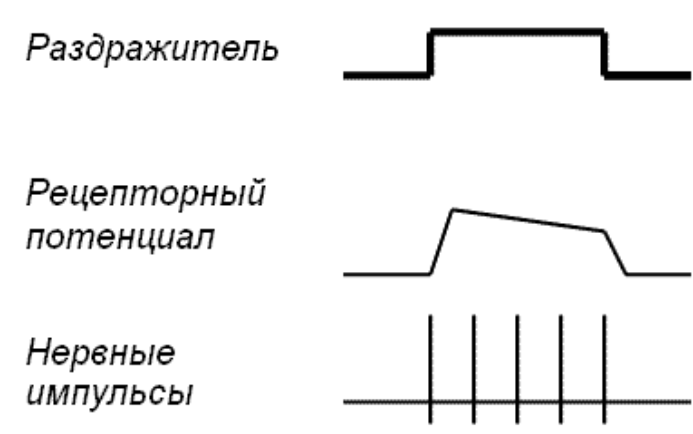

\section{Сильный раздражитель}
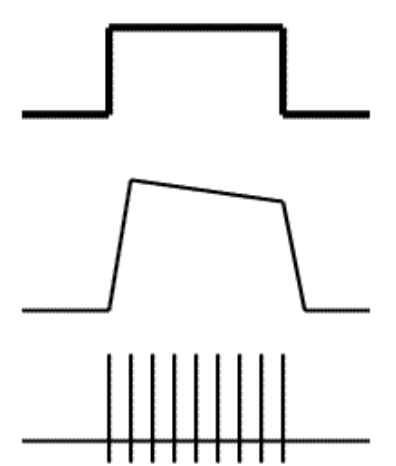

Рис. 1. Преобразование энергии внешнего раздражителя в рецепторный потенциал и серию нервных импульсов при действии слабого и сильного раздражителя 
не представляет интереса, поскольку характеризует сухой эпителий, сигнализирующий отсутствие излишков тепла в организме. Значение сопротивления кожи меньше 75 Ом с обильным выделением пота и интенсивностью нагрева, близкой к болевым ощущениям, может служить верхним предельным диапазоном интенсивности нагрева.

Экспериментально полученная активная область сопротивлений кожного покрова лежит в диапазоне 500-200 Ом. Центр этой зоны отличается как для различных людей, так и для мест считывания данных на теле. Для обеспечения устойчивости управления в активной области использовался алгоритм знаков с изменением содержимого регистра управления на единицу после каждого такта и формирования среднего выборки. Кроме резистивных, существуют датчики оптоэлектронного или емкостного типов, которые определяют момент активизации потовых желез, например [14], что может служить опорной точкой $m_{0}$.

Левый лепесток управления в диапазоне $(0,5-19)$ КОм меняет содержимое на 2, в диапазоне (10-100) КОм на 3, в диапазоне $(100-1000)$ КОм на 4, выше 1 МОм на 5. Соответственно, в правом лепестке значения меняются в диапазоне (200-150) Ом на 2, в диапазоне (150-100) Ом - на 3 , в диапазоне (100-75) - на 4, меньше 75 Ом - на 5. Следовательно, динамика формирования управ- ляющих воздействий в центральной зоне и границах управления существенно отличается.

В качестве принципа управления инфракрасными излучателями выбрано широтно-импульсное симисторное управление, предусмотренное в ARDUINO. Параллельно включенные инфракрасные керамические, трубчатые и ламповые инфракрасные излучатели имели постоянные времени 8 минут, 4 минуты, 2 секунды соответственно. Мощности излучателей различных спектральных диапазонов примерно одинаковые, диапазон рабочих интенсивностей терапевтического диапазона составлял $60-90 \%$ от максимального значения, камера предварительно прогревалась на уровне $50 \%$ мощности в течение 10 минут. Период измерения кожного сопротивления - 2 сек, количество уровней управления - 256 , время терапевтической процедуры 20-25 минут.

Выводы. Показано, что для инфракрасной системы регулирования с биологической обратной связью предпочтительна обработка первичной признаковой информации методами статистики нечисловых данных, снижающих влияние неопределенности и неоднозначности на результаты принятия решения.

Предложен алгоритм повышения динамических характеристик системы управления инфракрасными излучателями, основанный на биологических аналогиях и нечеткой логике.

\section{Список литературы:}

1. Деклараційний патент № 58051А (Україна). Спосіб пелоїдотерапії та камера для його здійснення / Косовєров С.О., Тищук М.М., Мещеряков В.І., Веселкова Т.О.

2. Федотов А.А., Акулов С.А. Измерительные преобразователи биомедицинских сигналов систем клинического мониторинга. Москва : Радио и связь. 2013. 250 с.

3. Гнатовская А.А., Мещеряков Д.В., Черепанова Е.В. Концепция преобразования данных инфракрасной системой с биологической обратной связью. Вчені записки ТНУ імені В.I. Вернадського. 2018. T. 29(68). № 2. С. 116-120.

4. Кухтичев А.А., Клёнов Е.А. Носимые устройства микроэлектроники как основа биологической обратной связи системы «ЦифроМед» в авиации и космонавтике. Врач и медицинские технологии. 2015. № 3. C. 39-48.

5. Filatova A.E. Nonlinear filtration of biomedical signals with the locally concentrated signs in task of structural identification. Herald of the National Technical University "KhPI". Subject issue: Information Science and Modelling. Kharkov : NTU “KhPI”. 2011. No. 17. Pp. 168-174.

6. Файнзильберг Л.С. ФАЗАГРАФ - эффективная информационная технология обработки ЭКГ в задаче скрининга ишемической болезни сердца. Клиническая информатика и телемедицина. 2010. Т. 6. Вып. 7. С. 22-30.

7. Калиниченко А.Н. Компьютерные методы автоматического анализа ЭКГ в системах кардиологического наблюдения : диссертация на соискание ученой степени доктора технических наук. Санкт-Петербург. 2008. 205 c.

8. Акулов С.А., Федотов А.А. Основы теории биотехнических систем. Москва : ФИЗМАТЛИТ. 2014. 259 с.

9. Пономарев А.С. Нечеткие множества в задачах автоматизированного управления и принятия решений. Харьков : НПУ «ХПИ». 2005. 232 с.

10. Каплан А.Я. ЭЭГ как управляющий сигнал: на пути к биотехнической нейрокоммуникации. Биоуправление: теория и практика. Новосибирск. 2010. С. 7-18. 
11. Орлов А.И. Нечисловая статистика. Москва : МЗ-Пресс. 2004. 513 с.

12. Батуев А.С. Физиология высшей нервной деятельности и сенсорных систем. Санкт-Петербург : Питер. 2010. 317 с.

13. Большаков А.А., Каримов Р.Н. Методы обработки многомерных данных и временных рядов. Москва : Горячая линия-Телеком. 2007. 522 с.

14. Платонов А.К. Высокочувствительный сенсор электрического сопротивления кожи человека. Препринты ИПМ им. М.В. Келдыша. 2012. № 18. 20 c. URL: http://library.keldysh.ru/preprint.asp?id=2012-18.

\section{Mescheryakov D.V. NON-NUMERICAL STATISTICAL DATA PROCESSING \\ IN A SYSTEM WITH BIOLOGICAL FEEDBACK}

The processing of output sign information in an infrared peloids therapy system with biological feedback, which can be used to treat the musculoskeletal system of ship crew members, is considered. The necessity of harmonizing the cybernetic and physiological description approaches used for technical and biological objects is substantiated. It is shown that the main problems encountered in processing the output feature signals of a biological object are their fuzziness and ambiguity. These features of the response of a physiological object to infrared exposure are due to both the complexity of signal conversion by the body and the imposition of accompanying signals on informative signals regarding the control of the irradiation process. The difficulty of obtaining an analytical relationship between the input effect and the response of the body, which can be fixed by non-invasive technical means, is substantiated. It is proposed to use non-numerical data statistics methods to describe output features. The features of processing high-quality data in relation to decision-making for managing a closed system for controlling the intensity of radiation are revealed. It is shown that the Kemeny average can serve as an indicator of sample values of primary data. A comparison of the sequence of average values of the moving sample allowed us to determine the decision-making mechanism regarding the direction of change in the intensity of the generated infrared radiation. The expediency of using the criterion of signs for making decisions in a system with biological feedback is substantiated. Achieving local goals of controlling the intensity of exposure to a biological object is reduced to the principle of tracking balancing, which is characterized by a high degree of resistance to abnormal emissions. To compensate for the disadvantage associated with the long time for entering the control mode, two ways to increase the dynamic characteristics of control are proposed. The first is based on non-numerical methods, by analogy with the operation of biological sensors in the physiological system, the other on fuzzy regulators.

Key words: biofeedback, non-digital statistics, decision making, management. 